\title{
Prolonged QT Interval in Neurodevelopmental Rat Model of Schizophrenia
}

\author{
Tibor Stracina ${ }^{1}$, Marina Ronzhina ${ }^{2}$, Tibor Stark ${ }^{3}$, Jana Ruda ${ }^{3}$, Eva Olsanska ${ }^{2}$, Petr Vesely ${ }^{2}$, \\ Vincenzo Micale ${ }^{4}$, Marie Novakova ${ }^{1}$ \\ ${ }^{1}$ Department of Physiology, Faculty of Medicine, Masaryk University, Brno, Czech Republic \\ ${ }^{2}$ Department of Biomedical Engineering, Brno University of Technology, Brno, Czech Republic \\ ${ }^{3}$ Department of Pharmacology, Faculty of Medicine, Masaryk University, Brno, Czech Republic \\ ${ }^{4}$ Centre for Neuroscience, Central European Institute of Technology, Masaryk University, Brno, \\ Czech Republic
}

\begin{abstract}
QT prolongation is an independent risk factor for development of ventricular arrhythmias and eventually sudden cardiac death. It is common side effect of various medications, including antipsychotics.

It was reported that schizophrenic patients treated with antipsychotics (e.g. haloperidol, olanzapine) manifested QT prolongation. Also, drug-free schizophrenic patients have increased $Q T$ interval compared to healthy controls. Possible mechanisms of QT prolongation connected with schizophrenia are still unclear.

The present study on isolated hearts clearly shows that rats with schizophrenia-like phenotype manifest significantly lengthened QT interval as compared to controls. This difference was further increased after acute haloperidol administration. These data indicate that methylazoxymethanol acetate rat model of schizophrenia may be applicable tool for studying of connection between schizophrenia and QT prolongation.
\end{abstract}

\section{Introduction}

Schizophrenia as one of the main indications for chronic antipsychotic treatment affects approximately $1 \%$ of the population worldwide and manifests at young adult age. Besides the main side effects of antipsychotics such as extrapyramidal symptoms and metabolic adverse effects, many patients suffer from cardiovascular complications, particularly lengthened QT interval [1]. QT prolongation increases the risk of ventricular arrhythmias and eventually sudden cardiac death [2].

Moreover, it was reported that also drug-free patients suffering from schizophrenia have an increased QT interval compared to age-matched healthy subjects [3]. It is supposed that the polymorphism in KCNH2 channel might be a possible connection between schizophrenia and QT prolongation. This channel is responsible for $\mathrm{K}^{+}$ delayed rectifier potassium current and its blocking (e.g. pharmacological or by a variant in the channel gene) leads to a significant QT prolongation. Nevertheless, the mechanism connecting schizophrenia with QT prolongation has not been fully elucidated yet. Moreover, studying of the reasons of QT lengthening in schizophrenic patients is rather complicated due to their frequent comorbities and certain incapability to cooperate. The need of suitable model for such studies is apparent.

Therefore, the present study aimed to evaluate heart rate (HR) and QT interval duration in methylazoxymethanol acetate rat model of schizophrenia. Basic electrogram parameters were studied in isolated hearts of rats with schizophrenia-like phenotype under resting conditions and after haloperidol administration.

\section{Methods}

The study was carried out according to the recommendations of the European Community Guide for the Care and Use of Laboratory Animals and according to the experimental protocol approved by the Committee on the Protection of Animals, Faculty of Medicine, Masaryk University.

\subsection{Experimental animals}

On gestational day 17, time-mated female albino Sprague-Dawley rats were injected intraperitoneally with methylazoxymethanol acetate (MAM, Midwest Research Institute, Kansas City, USA; MAM group) or vehicle (saline; CON group), as previously described [4]. The offspring were weaned on the postnatal day 22 and housed in groups of two or three littermates under controlled environmental conditions (relative humidity $50-60 \%$, temperature $23^{\circ} \mathrm{C} \pm 1^{\circ} \mathrm{C}$, inverted 12 -hour lightdark cycle) with food and water available $a b$ libitum. 
Only male offspring were used in the study.

The offspring of MAM-treated females (MAM group) manifested schizophrenia-like phenotype, which was evaluated by set of standard behavioural tests in adulthood.

\subsection{Isolated heart preparation}

For isolated heart experiment, 32 weeks old rats with body mass $547 \pm 68$ g were used. Nine schizophrenia-like (MAM) and six control (CON) rats were deeply anaesthetised. The heart was rapidly excised from the thorax, placed in a cold $\left(4^{\circ} \mathrm{C}\right)$ Krebs-Henseleit (K-H) solution and prepared for cannulation. The aorta was cannulated and the heart was perfused according to Langendorff at constant perfusion pressure (75 $\mathrm{mmHg}$ ) with K-H solution ( $\mathrm{NaCl}, 118 \mathrm{mM}$; $\mathrm{NaHCO}_{3}, 27 \mathrm{mM}$; $\mathrm{KCl}, 4.8 \mathrm{mM}$; $\mathrm{KH}_{2} \mathrm{PO}_{4}, 1.0 \mathrm{mM}$; $\mathrm{MgSO}_{4}, 1.2 \mathrm{mM}$; $\mathrm{CaCl}_{2}$, $1.2 \mathrm{mM}$; glucose, $10 \mathrm{mM} ; 37^{\circ} \mathrm{C}$; $\mathrm{pH} 7.4$ ) aerated with $95 \% \mathrm{O}_{2}$ and $5 \% \mathrm{CO}_{2}$. The isolated heart was allowed to stabilize for 20 minutes. Immediately after stabilization, experimental protocol continued with two 40-minuteslasting consecutive phases: haloperidol exposure and washout with $\mathrm{K}-\mathrm{H}$ solution. Haloperidol was administered diluted in $\mathrm{K}-\mathrm{H}$ solution at the concentration of $10 \mathrm{nmol} / \mathrm{L}$.

\subsection{Data acquisition}

Six Ag-AgCl electrodes placed in the inner surface of heart chamber were used for continual recording of electrogram in three orthogonal leads. Besides electrogram, the perfusion pressure and temperature of the perfusion solution were continuously monitored. All signals were recorded by an acquisition card NI USB6229 BNC (National Instruments, TX, USA) with sampling frequency of $5 \mathrm{kHz}$ and 12-bit resolution.

\subsection{Data analyses}

The whole processing was made in off-line mode using MATLAB R2014b (MathWorks, MA, USA). The positions of $\mathrm{R}$ peaks were detected by automatic detection method based on wavelet transform. HR was measured from the window of 10 consecutive RR intervals at the end of each experimental phase. In the same window, QT intervals were detected manually and corrected according to Bazett's formula.

Additionally, incidence of arrhythmias was assessed in electrograms. The total number of ventricular premature beats (VPBs) and supraventricular premature beats (SVPBs) was calculated for each experimental phase separately.

Basic descriptive statistic was then applied and results were expressed as mean \pm SD. According to the results of
Shapiro-Wilk test, calculated parameters are not normally distributed. Therefore, the non-parametric Mann-Whitney $\mathrm{U}$ test was used to test the differences between MAM and CON groups. Differences between data from consecutive phases were then verified using Wilcoxon signed-rank test. Spearman's rho was used to assess the correlation between numbers of pathological beats in consecutive experimental phases. For all above tests, $\mathrm{p}<0.05$ was considered significant.

\section{Results}

\subsection{Heart rate}

The values of HR are represented as box plots in Fig 1. Slightly higher HR values were found in the MAM group in comparison with that of CON group in all experimental phases $(270 \pm 10 \mathrm{bpm}$ in CON and $290 \pm 31 \mathrm{bpm}$ in MAM, respectively). However, no significant difference in HR between the groups was proven.

Administration of haloperidol as well as washout caused no significant changes of HR in either group (in comparison with HR measured in stabilization phase).

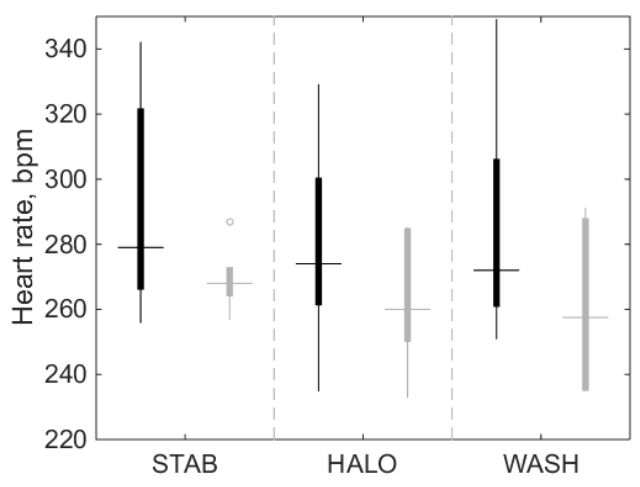

Figure 1. Heart rate of isolated hearts at the end of stabilization (STAB), haloperidol administration (HALO) and washout (WASH) measured in MAM (black) and CON (grey) groups. Displayed as median, the edges of the box indicate $25^{\text {th }}-75^{\text {th }}$ percentiles, circles indicate outliers.

\subsection{QTc interval}

Distribution of QTc values calculated in MAM and CON groups at the end of each experimental phase and results of statistical tests are presented in Fig. 2. It is obvious that MAM hearts have significantly longer QTc interval than CON at the end of stabilization $(64.8 \pm 1.9$ $\mathrm{ms}$ in CON and $69.5 \pm 4.8 \mathrm{~ms}$ in MAM, respectively; $\mathrm{p}<0.05$ ). This difference was even more significant during haloperidol administration because of increase of QTc in MAM and no change of QTc in CON group (63.2 \pm 2.5 
ms in CON and $72.7 \pm 5.4 \mathrm{~ms}$ in MAM, respectively; $\mathrm{p}<0.01)$. The parameter values remain significantly different in washout, too.

Due to high dispersion of the values, increase of QTc during haloperidol administration in MAM group was not significant. During washout, QTc in MAM was decreased at the level of stabilization value, while in CON was stable.

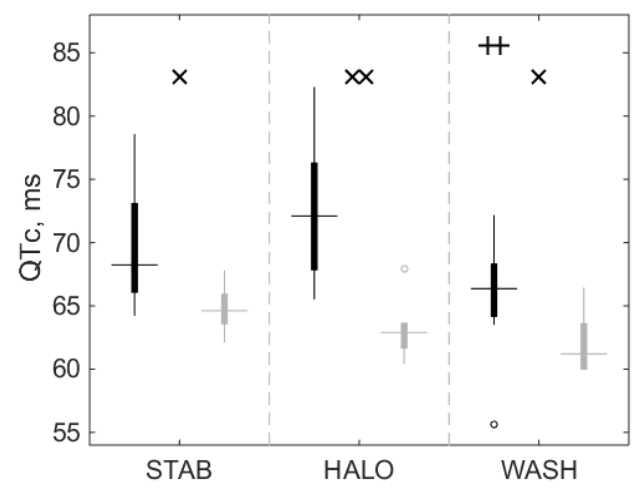

Figure 2. QTc interval at the end of stabilization (STAB), haloperidol administration (HALO) and washout (WASH) calculated in MAM (black) and CON (grey) group. Displayed as median, the edges of the box indicate $25^{\text {th }}-75^{\text {th }}$ percentiles, circles indicate outliers. ' $x$ ' and ' $x x^{\prime}$ indicate significant difference between the groups ( $\mathrm{p}<0.05$ and $\mathrm{p}<0.01$, respectively), ' ++ ' indicates significant difference between consecutive phases within the group $(\mathrm{p}<0.01)$.

\subsection{Incidence of arrhythmias}

VPBs were observed in electrograms of both groups only sporadically, except of two experiments from MAM group, where total number of VPBs in washout reached 5 and 48 , respectively.

Percentage distribution of SVPBs through the whole experiments within particular group is shown in Fig. 3. It is evident that the character of distribution is the same in both groups.

If both groups are analysed together, than more than 1 SVPB were found in $13 \%, 67 \%$ and $80 \%$ of total number of experiments in stabilization, haloperidol administration and washout, respectively. More than 10 SVPBs were observed in $20 \%$ and $33 \%$ of experiments in the second and last experimental phase, respectively. Significant positive correlation (Spearman's rho $=0.74, \mathrm{p}<0.005$ ) was obtained between total number of SVPBs during haloperidol administration and washout.

There is no difference in total number of SVPBs between MAM and CON groups, as can be seen in Fig. 4 . Number of SVPBs in MAM increases significantly during haloperidol administration and washout in comparison with corresponding previous phases.

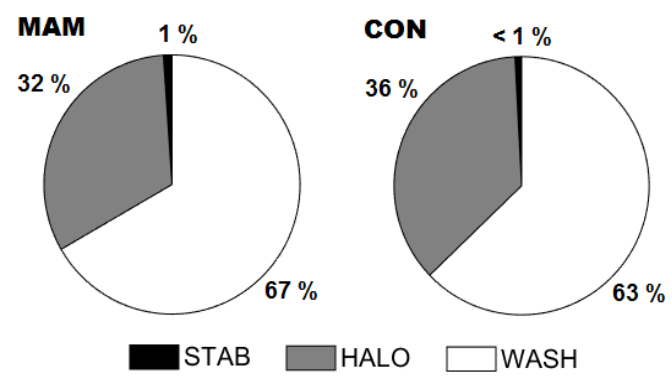

Figure 3. Percentage distribution of SVPBs through all experiments. STAB - stabilization, HALO - haloperidol administration, WASH - washout.

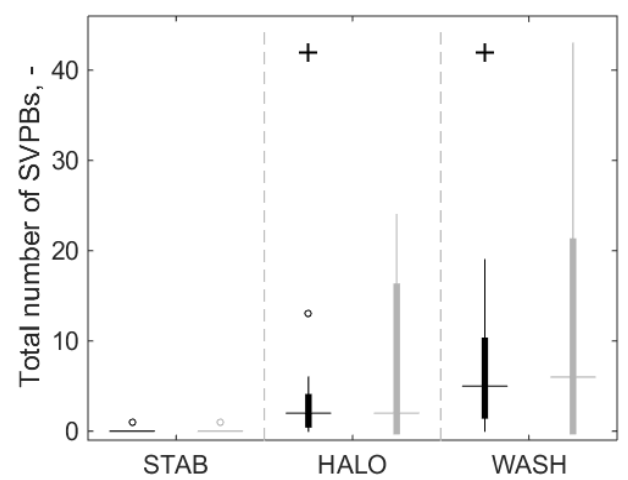

Figure 4. Distribution of total number of SVPBs during stabilization (STAB), haloperidol administration (HALO) and washout (WASH) in MAM (black) and CON (grey) group. Displayed as median, the edges of the box indicate $25^{\text {th }}-75^{\text {th }}$ percentiles, circles indicate outliers. ' + ' indicates significant difference between consecutive phases within the group $(\mathrm{p}<0.05)$.

\section{Discussion}

Based on the neurodevelopmental hypothesis of schizophrenia, it becomes increasingly applicable that an injection of the MAM in pregnant rat dams at gestational day 17 produces long lasting behavioral (i.e. sensorimotor gating deficits, hyper-locomotion, social behavior impairment, and cognitive deficits) and molecular (i.e.: dysregulation of dopaminergic, glutamatergic and GABAergic neurotransmission) changes in the offspring. These changes are consistent with those observed in schizophrenic patients [5].

According to the present data, MAM rats exhibit longer QT interval than controls. Moreover, QT prolongation is increased after administration of classical antipsychotic drug haloperidol.

One of the possible mechanisms of QT prolongation in schizophrenia-like rats might be the existence of variant polymorphism or mutation in gene for $\mathrm{KCNH} 2$ potassium channel [3] or in other genes (e.g. hERG gene) related to 
potassium channels and their regulation. Other mechanisms might be associated with alteration of dopamine D2 receptor signalling in the heart or with imbalance of autonomic nervous system, particularly with reduced efferent vagal activity [6] leading to abnormalities in heart rate variability [7].

Some of the abovementioned mechanisms may be common also for antipsychotic action. It has been repeatedly reported that antipsychotics affect hERG gene, which was set as a link between inherited and acquired QT prolongation [8]. Moreover, antipsychotics such as haloperidol have affinity to various receptor systems and may affect heart functions directly. The main effectiveness of antipsychotics against positive symptoms of schizophrenia lies in the pharmacological blockade of D2 receptors in the dopamine pathways of the brain. These receptors' signaling pathway includes inositol 1,4,5-trisphosphate receptors; thus, calcium concentration both in endoplasmic reticulum and cytosol can be altered [9]. Dopamine D2 receptors are also present in the heart. Therefore it might be presumed that antipsychotic treatment affects calcium handling in the cardiac cells. This may lead to changes of electric activity of the heart.

Still, the description of precise mechanisms of QT prolongation associated with schizophrenia and antipsychotic treatment need further investigation. Research on the highly translational neurodevelopmental MAM rat model of schizophrenia can bring new insights into these mechanisms.

\section{Acknowledgements}

This report was written at Masaryk university as part of the projects "Kardiovaskulární systém: od modelu pres terapii k prevenci" number MUNI/A/1365/2015 and "Experimentální farmakologický vývoj v neuropsychiatrii a onkologii“ number MUNI/A/1284/2015 with the support of the Specific University Research Grant, as provided by the Ministry of Education, Youth and Sports of the Czech Republic in the year 2016. This work was also supported by the grant project of the Czech Science Foundation GAP102/12/2034.

This study was co-financed from the SoMoPro II programme. The research leading to this invention has acquired a financial grant from the People Programme (Marie Curie action) of the Seventh Framework Programme of EU according to the REA Grant Agreement
No. 291782. The research is further co-financed by the South-Moravian Region. The study reflects only the author's views and that the Union is not liable for any use that may be made of the information contained therein.

\section{References}

[1] Beach SR, Celano CM, Noseworthy PA, Januzzi JL, Huffman JC. QTc prolongation, torsades de pointes, and psychotropic medications. Psychosomatics 2013;54:1-13.

[2] Wu CS, Tsai YT, Tsai HJ. Antipsychotic drugs and the risk of ventricular arrhythmia and/or sudden cardiac death: a nation-wide case-crossover study. J Am Heart Assoc 2015;4:e001568.

[3] Fujii K, Ozeki Y, Okayasu H, Takano Y, Shinozaki T, Hori H, Orui M, Horie M, Kunugi H, Shimoda K. QT is longer in drug-free patients with schizophrenia compared with age-matched healthy subjects. PLoS One 2014;9:e98555.

[4] Moore H, Jentsch JD, Ghajarnia M, Geyer MA, Grace AA. A neurobehavioral systems analysis of adult rats exposed to methylazoxymethanol acetate on E17: implications for the neuropathology of schizophrenia. Biol Psychiatry 2006;60:253-64.

[5] Lodge DJ, Grace AA. Gestational methylazoxymethanol acetate administration: a developmental disruption model of schizophrenia. Behav Brain Res 2009;204:306-12.

[6] Bär KJ. Cardiac Autonomic Dysfunction in Patients with Schizophrenia and Their Healthy Relatives - A Small Review. Front Neurol 2015;6:139.

[7] Montaquila JM, Trachik BJ, Bedwell JS. Heart rate variability and vagal tone in schizophrenia: A review. J Psychiatr Res 2015;69:57-66.

[8] Sanguinetti MC, Jiang C, Curran ME, Keating MT. A mechanistic link between an inherited and an acquired cardiac arrhythmia: HERG encodes the IKr potassium channel. Cell 1995;81:299-307.

[9] Beaulieu JM, Espinoza S, Gainetdinov RR. Dopamine Receptors - IUPHAR Review 13. Br J Pharmacol 2015;172:1-23.

Address for correspondence.

Tibor Stracina

Department of Physiology

Faculty of Medicine

Masaryk University

Kamenice 5, 62500 Brno, Czech Republic

stracina@med.muni.cz 